\title{
Management der Stapeslaserchirurgie - Wieviel Tage stationäre Behandlung sind erforderlich? Ergebnisse von 96 Operationen aus 12 Monaten
}

\author{
0. Ebeling, K. Frese, S. Fötzsch \\ Klinik für Hals-Nasen- \\ Ohrenheilkunde, \\ Plastische Operationen, \\ Spezielle HNO-Chirurgie des \\ Klinikum Lahr-Ettenheim \\ Klostenstr. 19 \\ 77933 Lahr \\ Tel 07821.932501 \\ Fax 07821.932064
}

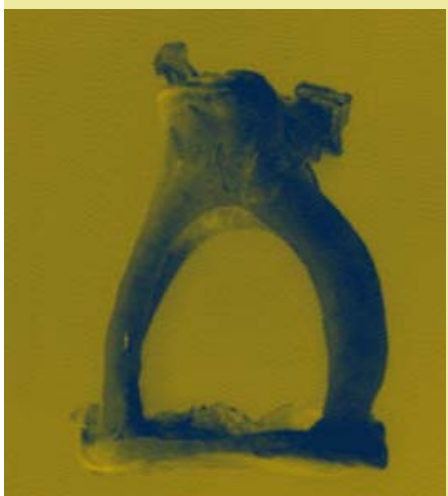

\section{Einleitung:}

Die Stapeschirurgie mit dem $\mathrm{CO}_{2}$ Laser ist als besonders innenohrschonendes Instrument etabliert. Unsere Fragestellung war, wieviel Tage stationäre Behandlung zum sicheren Management dieser Operation notwendig sind. Gerade unter dem Aspekt der DRG-Abrechnung spielt hier die Abwägung von medizinischer Notwendigkeit und Wirtschaftlichkeit eine größere Rolle denn je.
Methode:

Verwandt wurden Platinteflonprothesen mit einem Pistondurchmesser von $0,6 \mathrm{~mm}$. Zeigte sich eine Schwindelsymptomatik, erfolgte zunächst die Teildetamponade, bei persistierenden Beschwerden wurde nach Stennert infundiert.

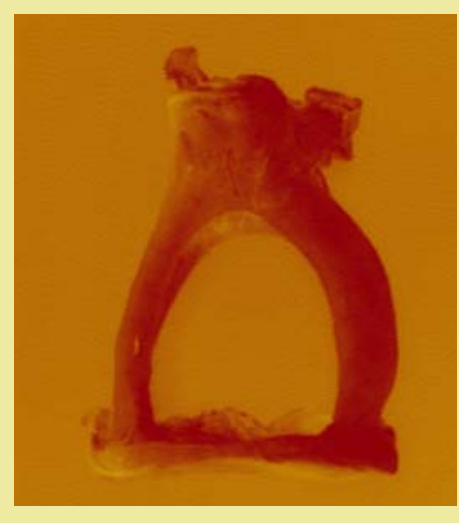

\section{Patienten und Methode:}

Bei insgesamt 96 Patienten wurde innerhalb von 12 Monaten eine Laserstapesplastik durchgeführt. Zeitraum 1.4.2003 - 1.4.2004. Wir operierten die 58 weiblichen und 38 männlichen Patienten mit dem Surgitouch $\mathrm{CO}_{2}$-LaserSystem der Firma Sharplan. Die Patienten wurden am zweiten postoperativen Tag untersucht, stellte sich bei stabiler Innenohrfunktion kein Nystagmus dar, erfolgte die Entlassung.

\section{Ergebnisse:}

Die durchschnittliche Schalleitungskomponente in der Summe der Frequenzen von $0,25-6 \mathrm{kHz}$ betrug $39 \mathrm{~dB}$ präoperativ, postoperativ lag sie bei $5,5 \mathrm{~dB}$.

$10,5 \%$ der Patienten berichtet am 2. Tage postoperativ über Schwindel, durch die Detamponade und das Infusionsschema über maximal 6 Tage konnten diese Beschwerden in allen Fällen folgenlos therapiert werden.

\section{Ergebnisse:}

Ein Ausfall der Innenohrfunktion trat in keinem Fall auf.

Keiner der symptomlos am zweiten Tage entlassenen Patienten entwickelte später eine vestibuläre oder cochleäre Symptomatik. Die durchschnittliche Behandlungszeit betrug insgesamt 2,6 Tage. Eine Revision mußte in keinem Fall erfolgen.

\section{Zusammenfassung:}

Unter der Voraussetzung von geübten Operateuren (in diesem Fall 3) erscheint das postoperative Kontrollsegment von zwei Tagen sicher und erfreulicherweise auch ideal im Bezug auf die DRGAbrechnung. 